Vol. 6, Issue 1, January 2019

\title{
Synthesis, Characterization and Antimicrobial Studies of Triazole - Thiazolidine Clubbed Heterocyclic Compounds
}

\author{
Nunna G Rameshbabu ${ }^{1}$, Dr.Sheetal Gulati ${ }^{2}$, Dr.H.S.Patel ${ }^{3}$ \\ Research Scholar, Rabindranath Tagore University, Bhopal (M.P.), India ${ }^{1}$ \\ Professor, Department of Chemistry, Rabindranath Tagore University, Bhopal (M.P.), India ${ }^{2}$ \\ Ex.Head and professor, Department of Chemistry, Sardar Patel University, Vallabh Vidyanagar (Gujarat), India ${ }^{3}$
}

\begin{abstract}
Amino-1,2,4-traizol (I) on condensation with 5-Phenyl substituted-2- furan carboxaldehyde (IIa-e) yield schiff bases namely $\mathrm{N}-[(5$-Arylfuran-2-yl)methylen]-4H-1,2,4-triazol-4-amine (IIIa-e). Each of schiff base on cyclization with Thioglycolic acid afforded 2-(5-Arylfuran-2-yl)-3-(4H-1,2,4-triazol-4-yl) thiazolidin-4-one (IVa-e). Following this mannich base reaction of each IVa-e with Formaldehyde and Piperidine give 2-(5-Arylfuran-2-yl)-5(piperidin-1-yl methyl)-3-(4H-1,2,4-triazol-4-yl)thiazolidin-4-one (Va-e). All the compounds of each series were characterized by spectral features and elemental contents. The compounds were also screened for their antimicrobial behavior.
\end{abstract}

Key words: Triazole, Schiff base, 4-Thiazolidine, Spectral Studies, Antibacterial and Antifungal Activities

\section{INTRODUCTION}

Recently considerable interest adopted to synthesis of 1,2,4-Triazole derivatives having prominent pharmaceutical activities $^{1-6} .4$-Thiazolidinones and their derivatives are also reported industrially for their antitubercular, antibacterial, antifungal, anticonvulsant activities ${ }^{7-8}$. One of the heterocyclic compound i.e. Furfural is an agricultural waste material have various reaction properties for production of polymers, drugs, dyes etc. ${ }^{9-12}$. The literature survey reveals that no such work reported in this direction, hence the present communication deals with the synthesis, characterization and antimicrobial activity of novel heterocyclic having 1,2,4-Traizol-4-thiazolidinone merged system. the work will be scanned in the following scheme. 
Vol. 6, Issue 1, January 2019<smiles>Nn1cnnc1</smiles>

4-Amino-1,2,4-triazole (1)

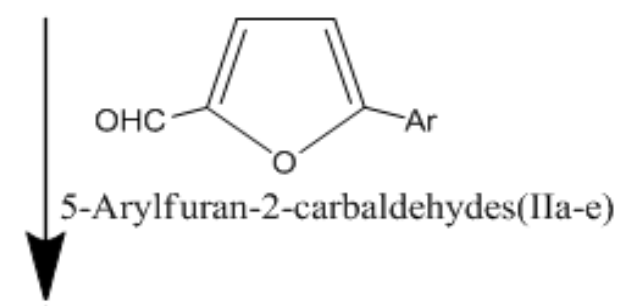<smiles>Brc1ccc(C=Nn2cnnc2)o1</smiles>

$N$-((5-arylfuran-2-yl)methylene)-4H-1,2,4-triazol-4-amine (IIIa-e)

thioglycolic acid<smiles>O=C1CSC(c2ccc(Br)o2)N1n1cnnc1</smiles>

2-(5-arylfuran-2-yl)-3-(4H-1,2,4-triazol-4-yl)thiazolidin-4-one (IVa-e)
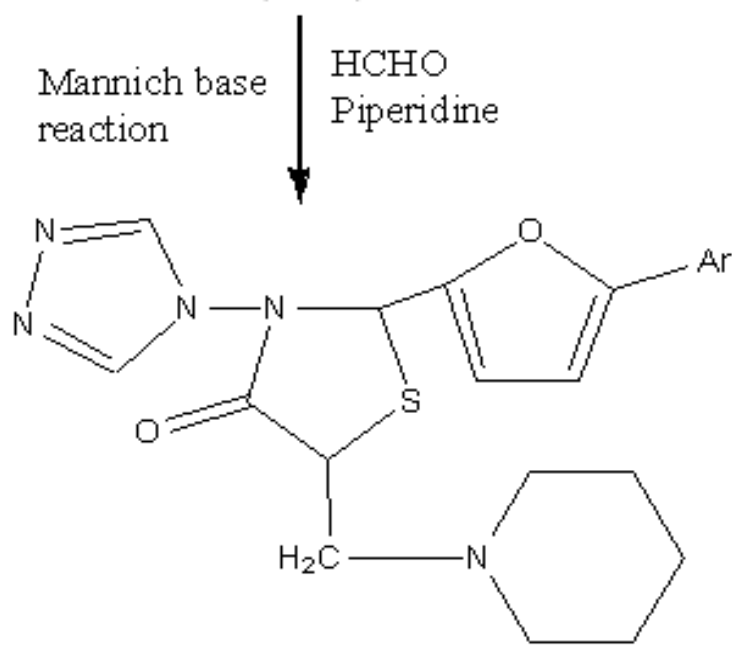

(Va-e)

Where $\mathrm{Ar}=$ (a) $\mathrm{C}_{6} \mathrm{H}_{5}$ (b) ${ }_{4}-\mathrm{ClC}_{6} \mathrm{H}_{4}$ (c) $4-\mathrm{BrC}_{6} \mathrm{H}_{4}$

(d) $4-\mathrm{NO}_{2} \mathrm{C}_{6} \mathrm{H}_{4}$ (e) $2,4-\mathrm{Cl}_{2} \mathrm{C}_{6} \mathrm{H}_{3}$ SCHEME-1 
Vol. 6, Issue 1, January 2019

\section{EXPERIMENTAL}

\section{Material and Methods}

The IR spectra were taken in $\mathrm{KBr}$ on a Nicolet 400D spectrometer and ${ }^{1} \mathrm{H}$ NMR spectra were scanned in DMSO solvent on a Bruker spectrometer at $400 \mathrm{MHz}$. LC-MS of all samples taken on LC-MSD-Trap-SL_01046. 5-Arylfuran-2carbaldehydes (IIa-e) were prepared according to reported method ${ }^{13}$. All other reagents used were of A.R.grade.

\section{Synthesis of N-[(5-Arylfuran-2-yl)methylene]-4H-1,2,4-triazol-4-amine (IIIa-e)}

4-Amino-1,2,4-triazole (I) (0.01mole) and the 5-Arylfuran-2-carbaldehydes (IIa-e) mixed in ethanol (25 ml) and refluxed for $6 \mathrm{hrs}$. The solid mass was filtered, washed by dry ether and air dried. The details of all these compounds are furnished in Table -1 .

Table:-1 Analytical Data Of Compounds (IIIA-E)

\begin{tabular}{|c|c|c|c|c|c|c|c|}
\hline \multirow{3}{*}{ Compd. } & \multirow{3}{*}{$\begin{array}{l}\text { Molecular formula } \\
\text { (Mol.wt.) }\end{array}$} & \multirow{3}{*}{$\begin{array}{l}\text { LC-MS } \\
\text { Data }\end{array}$} & \multirow{3}{*}{ Yield \% } & \multirow{3}{*}{$\underset{{ }^{0} \mathrm{C}}{\text { M.P.* }}$} & \multicolumn{3}{|c|}{ Elemental Analysis } \\
\hline & & & & & $\% \mathrm{C}$ & $\% \mathrm{H}$ & $\% \mathrm{~N}$ \\
\hline & & & & & $\begin{array}{l}\text { Found } \\
\text { (Calcd.) }\end{array}$ & $\begin{array}{l}\text { Found } \\
\text { (Calcd.) }\end{array}$ & $\begin{array}{l}\text { Found } \\
\text { (Calcd.) }\end{array}$ \\
\hline IIIa & $\begin{array}{c}\mathrm{C}_{13} \mathrm{H}_{10} \mathrm{~N}_{4} \mathrm{O} \\
(238)\end{array}$ & 240 & 85 & 196-197 & $\begin{array}{c}65.5 \\
(65.54)\end{array}$ & $\begin{array}{c}4.2 \\
(4.23)\end{array}$ & $\begin{array}{c}23.5 \\
(23.52)\end{array}$ \\
\hline IIIb & $\begin{array}{c}\mathrm{C}_{13} \mathrm{H}_{9} \mathrm{~N}_{4} \mathrm{OCl} \\
(272)\end{array}$ & 285 & 82 & $202-204$ & $\begin{array}{c}57.2 \\
(57.26) \\
\end{array}$ & $\begin{array}{c}3.3 \\
(3.33)\end{array}$ & $\begin{array}{c}20.5 \\
(20.55)\end{array}$ \\
\hline IIIc & $\begin{array}{c}\mathrm{C}_{13} \mathrm{H}_{9} \mathrm{~N}_{4} \mathrm{OBr} \\
(317)\end{array}$ & 329 & 78 & 198-199 & $\begin{array}{c}49.2 \\
(49.23)\end{array}$ & $\begin{array}{c}2.8 \\
(2.86)\end{array}$ & $\begin{array}{c}17.6 \\
(17.67)\end{array}$ \\
\hline IIId & $\begin{array}{c}\mathrm{C}_{13} \mathrm{H}_{9} \mathrm{~N}_{5} \mathrm{O}_{3} \\
(283)\end{array}$ & 297 & 75 & 204-206 & $\begin{array}{c}55.1 \\
(55.13)\end{array}$ & $\begin{array}{c}3.1 \\
(3.20)\end{array}$ & $\begin{array}{c}24.7 \\
(24.73)\end{array}$ \\
\hline IIIe & $\begin{array}{c}\mathrm{C}_{13} \mathrm{H}_{8} \mathrm{~N}_{4} \mathrm{OCl}_{2} \\
(307)\end{array}$ & 327 & 79 & 197-199 & $\begin{array}{c}50.8 \\
(50.84)\end{array}$ & $\begin{array}{c}2.6 \\
(2.63)\end{array}$ & $\begin{array}{c}18.2 \\
(18.24)\end{array}$ \\
\hline
\end{tabular}

* Uncorrected

\section{Synthesis of 2-(5-Arylfuran-2-yl)-3-(4H-1,2,4-triazol-4-yl)thiazolidin-4-one (IVa-e)}

The compounds (IIIa-e) $(0.01$ mole) in Tetrahydrofuran $(40 \mathrm{ml})$ and Thioglycolic acid $(0.01 \mathrm{~mole})$ with a $20 \mathrm{mg}$ of anhydrous $\mathrm{ZnCl}_{2}$ was heated an at $100^{\circ} \mathrm{C}$ for $10 \mathrm{hrs}$. The solvent was removed, the residue obtained was dissolved in toluene and passed through a column of silica gel using toluene: chloroform (7:3 v/v) mixture as an eluent. The eluate was concentrated and the product crystallized from isopropanol to get 4-Thiazolidinones (IVa-e). The details of these compounds are presented in table-2.

Table:-2 Analytical Data of Compounds (IVa-e)

\begin{tabular}{|c|c|c|c|c|c|c|c|c|}
\hline \multirow{3}{*}{ Compd. } & \multirow{3}{*}{$\begin{array}{c}\text { Molecular formula } \\
\text { (Mol.wt.) }\end{array}$} & \multirow{3}{*}{$\begin{array}{l}\text { LC-MS } \\
\text { Data }\end{array}$} & \multirow{3}{*}{ Yield \% } & \multirow{3}{*}{$\underset{{ }^{\mathbf{M}} \mathrm{C}}{\mathrm{M} .}$} & \multicolumn{3}{|c|}{ Elemental Analysis } & \multirow[b]{2}{*}{$\% \mathrm{~S}$} \\
\hline & & & & & $\% \mathrm{C}$ & $\% \mathrm{H}$ & $\% \mathbf{N}$ & \\
\hline & & & & & $\begin{array}{l}\text { Found } \\
\text { (Calcd.) }\end{array}$ & $\begin{array}{l}\text { Found } \\
\text { (Calcd.) }\end{array}$ & $\begin{array}{l}\text { Found } \\
\text { (Calcd.) }\end{array}$ & $\begin{array}{l}\text { Found } \\
\text { (Calcd.) }\end{array}$ \\
\hline IVa & $\begin{array}{c}\mathrm{C}_{15} \mathrm{H}_{12} \mathrm{~N}_{4} \mathrm{O}_{2} \mathrm{~S} \\
(312)\end{array}$ & 314 & 68 & $231-232$ & $\begin{array}{c}57.6 \\
(57.68)\end{array}$ & $\begin{array}{c}3.8 \\
(3.87)\end{array}$ & $\begin{array}{c}17.9 \\
(17.94)\end{array}$ & $\begin{array}{c}10.2 \\
(10.27)\end{array}$ \\
\hline $\mathrm{IVb}$ & $\begin{array}{c}\mathrm{C}_{15} \mathrm{H}_{11} \mathrm{~N}_{4} \mathrm{O}_{2} \mathrm{SCl} \\
(346)\end{array}$ & 329 & 62 & $214-215$ & $\begin{array}{c}51.9 \\
(51.95)\end{array}$ & $\begin{array}{c}3.1 \\
(3.20)\end{array}$ & $\begin{array}{c}16.0 \\
(16.16)\end{array}$ & $\begin{array}{c}9.2 \\
(9.25)\end{array}$ \\
\hline IVc & $\begin{array}{c}\mathrm{C}_{15} \mathrm{H}_{11} \mathrm{~N}_{4} \mathrm{O}_{2} \mathrm{SBr} \\
(391)\end{array}$ & 406 & 63 & $226-228$ & $\begin{array}{c}46.0 \\
(46.05)\end{array}$ & $\begin{array}{c}2.8 \\
(2.83)\end{array}$ & $\begin{array}{c}14.3 \\
(14.32)\end{array}$ & $\begin{array}{c}8.1 \\
(8.20)\end{array}$ \\
\hline IVd & $\begin{array}{c}\mathrm{C}_{15} \mathrm{H}_{11} \mathrm{~N}_{5} \mathrm{O}_{4} \mathrm{~S} \\
(357)\end{array}$ & 371 & 68 & $219-220$ & $\begin{array}{c}50.4 \\
(50.42)\end{array}$ & $\begin{array}{c}3.0 \\
(3.10)\end{array}$ & $\begin{array}{c}19.5 \\
(19.60)\end{array}$ & $\begin{array}{c}8.9 \\
(8.97)\end{array}$ \\
\hline IVe & $\begin{array}{c}\mathrm{C}_{15} \mathrm{H}_{10} \mathrm{~N}_{4} \mathrm{O}_{2} \mathrm{SCl}_{2} \\
(381)\end{array}$ & 395 & 63 & $224-226$ & $\begin{array}{c}47.2 \\
(47.26) \\
\end{array}$ & $\begin{array}{c}2.6 \\
(2.64) \\
\end{array}$ & $\begin{array}{c}14.6 \\
(14.70) \\
\end{array}$ & $\begin{array}{c}8.3 \\
(8.41) \\
\end{array}$ \\
\hline
\end{tabular}

*Uncorrected

Mannich base formation of 2-(5-Arylfuran-2-yl)-5-(piperidin-1-yl methyl)-3-(4H-1,2,4-triazol-4-yl) thiazolidin-4one (Va-e)

The each of above 4-Thiazolidinone derivatives (IVa-e) was refluxed with piperidine and formaldehyde $(\approx 37 \%$ w/w) solutions at stoichiometric ratio in 1,4-dioxane. The product was filtered, washed by ethanol and air-dried. The analytical data are given in Table-3. 
Vol. 6, Issue 1, January 2019

Table:-3 Analytical Data of Compounds (Va-e)

\begin{tabular}{|c|c|c|c|c|c|c|c|c|}
\hline \multirow{3}{*}{ Compd. } & \multirow{3}{*}{$\begin{array}{l}\text { Molecular formula } \\
\text { (Mol.wt.) }\end{array}$} & \multirow{3}{*}{$\begin{array}{c}\text { LC-MS } \\
\text { Data }\end{array}$} & \multirow{3}{*}{ Yield \% } & \multirow{3}{*}{$\underset{{ }^{\mathbf{M}} \mathbf{C}}{\text { M.P.* }}$} & \multicolumn{3}{|c|}{ Elemental Analysis } & \multirow[b]{2}{*}{$\% \mathrm{~S}$} \\
\hline & & & & & $\% \mathrm{C}$ & $\% \mathrm{H}$ & $\% \mathbf{N}$ & \\
\hline & & & & & $\begin{array}{l}\text { Found } \\
\text { (Calcd.) }\end{array}$ & $\begin{array}{l}\text { Found } \\
\text { (Calcd.) }\end{array}$ & $\begin{array}{l}\text { Found } \\
\text { (Calcd.) }\end{array}$ & $\begin{array}{l}\text { Found } \\
\text { (Calcd.) }\end{array}$ \\
\hline $\mathrm{Va}$ & $\begin{array}{c}\mathrm{C}_{21} \mathrm{H}_{23} \mathrm{~N}_{5} \mathrm{O}_{2} \mathrm{~S} \\
(409)\end{array}$ & 422 & 70 & $180-181$ & $\begin{array}{c}61.5 \\
(61.59)\end{array}$ & $\begin{array}{c}5.6 \\
(5.66)\end{array}$ & $\begin{array}{c}16.9 \\
(17.10)\end{array}$ & $\begin{array}{c}7.8 \\
(7.83)\end{array}$ \\
\hline $\mathrm{Vb}$ & $\begin{array}{c}\mathrm{C}_{21} \mathrm{H}_{22} \mathrm{~N}_{5} \mathrm{O}_{2} \mathrm{SCl} \\
(443)\end{array}$ & 458 & 65 & $196-198$ & $\begin{array}{c}56.8 \\
(56.81) \\
\end{array}$ & $\begin{array}{c}4.9 \\
(4.99) \\
\end{array}$ & $\begin{array}{c}15.7 \\
(15.78)\end{array}$ & $\begin{array}{c}7.2 \\
(7.22) \\
\end{array}$ \\
\hline $\mathrm{Vc}$ & $\begin{array}{c}\mathrm{C}_{21} \mathrm{H}_{22} \mathrm{~N}_{5} \mathrm{O}_{2} \mathrm{SBr} \\
(488)\end{array}$ & 503 & 66 & $203-204$ & $\begin{array}{c}51.6 \\
(51.64) \\
\end{array}$ & $\begin{array}{c}4.5 \\
(4.54) \\
\end{array}$ & $\begin{array}{c}14.3 \\
(14.34)\end{array}$ & $\begin{array}{c}6.5 \\
(6.57) \\
\end{array}$ \\
\hline $\mathrm{Vd}$ & $\begin{array}{c}\mathrm{C}_{21} \mathrm{H}_{22} \mathrm{~N}_{6} \mathrm{O}_{4} \mathrm{~S} \\
(454)\end{array}$ & 468 & 64 & $211-212$ & $\begin{array}{c}55.4 \\
(55.49) \\
\end{array}$ & $\begin{array}{c}4.8 \\
(4.88) \\
\end{array}$ & $\begin{array}{c}14.0 \\
(14.08)\end{array}$ & $\begin{array}{c}7.0 \\
(7.05) \\
\end{array}$ \\
\hline $\mathrm{Ve}$ & $\begin{array}{c}\mathrm{C}_{21} \mathrm{H}_{21} \mathrm{~N}_{5} \mathrm{O}_{2} \mathrm{SCl}_{2} \\
(477)\end{array}$ & 495 & 61 & $215-217$ & $\begin{array}{c}52.7 \\
(52.72)\end{array}$ & $\begin{array}{c}4.4 \\
(4.42)\end{array}$ & $\begin{array}{c}14.6 \\
(14.64)\end{array}$ & $\begin{array}{c}6.6 \\
(6.70)\end{array}$ \\
\hline
\end{tabular}

*Uncorrected

III.

BIOLOGICAL SCREENING

\section{Antibacterial activities}

The antibacterial activities of all the compounds were studied against gram-positive bacteria (Staphylococcus aureus and Bacillus subtilis) and gram-negative bacteria (E.coli, and klebsiella promioe) at a concentration of 50 $\mu \mathrm{g} / \mathrm{ML}$ by agar cup plate method ${ }^{14}$. A methanol system was used as control in this method. Similar conditions using tetracycline as a control was used standard for comparison. The results in terms of percentage area of inhibition growth of bacteria are given in table- 4 to 6 . The results show that compounds IIIe, IVe and Ve are more toxic for bacteria.

Table:-4 Antibacterial Activity of Compounds (IIIa-e)

\begin{tabular}{|c|c|c|c|c|}
\hline \multirow{2}{*}{ Compounds } & \multicolumn{2}{|c|}{ Gram +Ve } & \multicolumn{2}{c|}{ Gram - Ve } \\
\cline { 2 - 5 } & $\begin{array}{c}\text { Staphylococcus } \\
\text { aureus }\end{array}$ & $\begin{array}{c}\text { Bacillus } \\
\text { subtilis }\end{array}$ & E.coli & $\begin{array}{c}\text { Klebsiella } \\
\text { promioe }\end{array}$ \\
\hline IIIa & 54 & 53 & 56 & 48 \\
\hline IIIb & 56 & 53 & 51 & 56 \\
\hline IIIc & 57 & 55 & 65 & 54 \\
\hline IIId & 63 & 58 & 59 & 53 \\
\hline IIIe & 68 & 67 & 77 & 61 \\
\hline
\end{tabular}

Table:-5 Antibacterial Activity of Compounds (IVa-e)

\begin{tabular}{|c|c|c|c|c|}
\hline \multirow{2}{*}{ Compounds } & \multicolumn{2}{|c|}{ Gram +Ve } & \multicolumn{2}{c|}{ Gram -Ve } \\
\cline { 2 - 5 } & $\begin{array}{c}\text { Staphylococcus } \\
\text { aureus }\end{array}$ & $\begin{array}{c}\text { Bacillus } \\
\text { subtilis }\end{array}$ & E.coli & $\begin{array}{c}\text { Klebsiella } \\
\text { promioe }\end{array}$ \\
\hline IVa & 56 & 54 & 58 & 51 \\
\hline IVb & 58 & 58 & 59 & 56 \\
\hline IVc & 60 & 59 & 67 & 54 \\
\hline IVd & 64 & 60 & 69 & 53 \\
\hline IVe & 71 & 72 & 77 & 66 \\
\hline
\end{tabular}

Table:-6 Antibacterial Activity of Compounds (Va-e)

\begin{tabular}{|c|c|c|c|c|}
\hline \multirow{2}{*}{ Compounds } & \multicolumn{2}{|c|}{ Gram +Ve } & \multicolumn{2}{c|}{ Gram -Ve } \\
\cline { 2 - 5 } & $\begin{array}{c}\text { Staphylococcus } \\
\text { aureus }\end{array}$ & $\begin{array}{c}\text { Bacillus } \\
\text { subtilis }\end{array}$ & E.coli & $\begin{array}{c}\text { Klebsiella } \\
\text { promioe }\end{array}$ \\
\hline Va & 58 & 55 & 59 & 53 \\
\hline Vb & 60 & 59 & 60 & 57 \\
\hline Vc & 61 & 61 & 68 & 55 \\
\hline Vd & 66 & 63 & 70 & 54 \\
\hline Ve & 73 & 74 & 78 & 68 \\
\hline
\end{tabular}




\section{International Advanced Research Journal in Science, Engineering and Technology}

Vol. 6, Issue 1, January 2019

\section{Antifungal activities}

The fungicidal activity of all the compounds was studied at $1000 \mathrm{ppm}$ concentration in vitro. Plant pathogenic organisms used were Nigrospora Sp, Aspergillus niger, Botrydepladia thiobromine, and Rhizopus nigricum, Fusarium oxyporium. The antifungal activity of all the compounds (IIIa-e), (IVa-e) and (Va-e) were measured on each of these plant pathogenic strains on a potato dextrose agar (PDA) medium. The PDA media were poured into sterile petri plates having $100 \mathrm{mg}$ sample, then the Petri dishes were kept inside for 5 days, 5 days culture (i.e. fungi) was inoculated into Petri plates. All the plates were kept aside per 5 days, the percentage inhibition for growth of fungi was calculated as follows

\section{Percentage of inhibition $=100(X-Y) / X$}

Where, $\mathrm{X}=$ Area of colony in control plate $\mathrm{Y}=$ Area of colony in test plate

The results of various compounds (IIIa-e), (IVa-e) and (Va-e) is shown in Tables-7,8 and 9.

Table:-7 Antifungal Activity of Compounds (IIIa-e)

\begin{tabular}{|c|c|c|c|c|c|}
\hline \multicolumn{7}{|c|}{ Zone of Inhibition of growth at 1000 ppm (\%) } \\
\hline Compounds & $\begin{array}{c}\text { Nigrospora } \\
\text { Sp. }\end{array}$ & $\begin{array}{c}\text { Aspergillus } \\
\text { Niger }\end{array}$ & $\begin{array}{c}\text { Botrydepladia } \\
\text { Thiobromine }\end{array}$ & $\begin{array}{c}\text { Rhizopus } \\
\text { Nigricum }\end{array}$ & $\begin{array}{c}\text { Fusarium } \\
\text { oxyporium }\end{array}$ \\
\hline IIIa & 56 & 50 & 61 & 55 & 67 \\
\hline IIIb & 66 & 67 & 63 & 62 & 68 \\
\hline IIIc & 67 & 66 & 69 & 61 & 66 \\
\hline IIId & 69 & 68 & 70 & 74 & 64 \\
\hline IIIe & 70 & 72 & 73 & 75 & 76 \\
\hline
\end{tabular}

Table:-8 Antifungal Activity of Compounds (IVa-e)

\begin{tabular}{|c|c|c|c|c|c|}
\hline \multicolumn{7}{|c|}{ Zone of Inhibition of growth at 1000 ppm (\%) } \\
\hline Compounds & $\begin{array}{c}\text { Nigrospora } \\
\text { Sp. }\end{array}$ & $\begin{array}{c}\text { Aspergillus } \\
\text { Niger }\end{array}$ & $\begin{array}{c}\text { Botrydepladia } \\
\text { Thiobromine }\end{array}$ & $\begin{array}{c}\text { Rhizopus } \\
\text { Nigricum }\end{array}$ & $\begin{array}{c}\text { Fusarium } \\
\text { oxyporium }\end{array}$ \\
\hline IVa & 59 & 53 & 62 & 57 & 69 \\
\hline IVb & 67 & 69 & 65 & 66 & 70 \\
\hline IVc & 70 & 67 & 71 & 64 & 69 \\
\hline IVd & 72 & 70 & 73 & 76 & 66 \\
\hline IVe & 72 & 74 & 75 & 77 & 79 \\
\hline
\end{tabular}

Table:-9 Antifungal Activity of Compounds(Va-e)

\begin{tabular}{|c|c|c|c|c|c|}
\hline \multicolumn{7}{|c|}{ Zone of Inhibition of growth at 1000 ppm (\%) } \\
\hline Compounds & $\begin{array}{c}\text { Nigrospora } \\
\text { Sp. }\end{array}$ & $\begin{array}{c}\text { Aspergillus } \\
\text { Niger }\end{array}$ & $\begin{array}{c}\text { Botrydepladia } \\
\text { Thiobromine }\end{array}$ & $\begin{array}{c}\text { Rhizopus } \\
\text { Nigricum }\end{array}$ & $\begin{array}{c}\text { Fusarium } \\
\text { oxyporium }\end{array}$ \\
\hline Va & 61 & 54 & 63 & 58 & 70 \\
\hline Vb & 68 & 70 & 67 & 67 & 72 \\
\hline Vc & 71 & 68 & 72 & 65 & 73 \\
\hline Vd & 74 & 71 & 74 & 78 & 67 \\
\hline Ve & 76 & 75 & 76 & 79 & 80 \\
\hline
\end{tabular}

\section{RESULTS AND DISCUSSION}

The 4-Amino-1,2,4-triazole (I) on reaction with 5-Arylfuran-2-carbaldehydes (IIa-e), perform schiff bases $\mathrm{N}$-[(5Arylfuran-2-yl)methylene]-4H-1,2,4-triazol-4-amine (IIIa-e). The structures of (IIIa-e) were confirmed by elemental analysis and IR spectra bands at 1625-1650 (C=N), 3040-3080 $\mathrm{cm}^{-1}\left(\mathrm{C}-\mathrm{H}\right.$, of Ar.),1185(C-O-C), 3385(-OH), $2850 \mathrm{~cm}^{-1}$ $\left(-\mathrm{OCH}_{3}\right), 2950,1370 \mathrm{~cm}^{-1}\left(-\mathrm{CH}_{3}\right), 1080(-\mathrm{Cl}), 1555,1375\left(-\mathrm{NO}_{2}\right)$ and the ${ }^{1} \mathrm{H}$ NMR signals $6.62-9.01(9 \mathrm{H}, \mathrm{m}, \mathrm{Ar}-\mathrm{H})$, 8.41-8.80 $(1 \mathrm{H}, \mathrm{s},-\mathrm{N}=\mathrm{CH})$. The $\mathrm{C}, \mathrm{H}, \mathrm{N}$ analysis data of all compounds are agree with desired structure.

Similarly 2-(5-Arylfuran-2-yl)-3-(4H-1,2,4-triazol-4-yl)thiazolidin-4-one (IVa-e) shows IR spectra bands at $1680 \mathrm{~cm}^{-1}$ $\left(\mathrm{C}=\mathrm{O}\right.$ of thiazolidinone ring), $720 \mathrm{~cm}^{-1}$ (C-S-C of thiazolidinone ring), $3080-3090 \mathrm{~cm}^{-1}\left(\mathrm{CH}_{2}\right.$ of thiazolidinone ring), $3040-3080 \mathrm{~cm}^{-1}$ (C-H, of Ar.), 3450-3560 cm-1 (-OH), 1185(C-O-C), 3385(-OH), $2850 \mathrm{~cm}^{-1}\left(-\mathrm{OCH}_{3}\right), 2950,1370 \mathrm{~cm}^{-1}$ $\left(-\mathrm{CH}_{3}\right), 1080(-\mathrm{Cl}), 1555,1375\left(-\mathrm{NO}_{2}\right)$ and ${ }^{1} \mathrm{H}$ NMR signal 3.80-3.90 (2H, s, $-\mathrm{CH}_{2}$ of the ring), 5.94-5.95 $(1 \mathrm{H}, \mathrm{s},-\mathrm{CH})$, 6.32-8.44 (9H, m, Ar - H). The C, H, N, S analysis data of all compounds are shown in Table-2. 
Vol. 6, Issue 1, January 2019

The Mannich base products were confirmed structurally by the elemental analysis, IR and NMR spectral features. The bands at $1680 \mathrm{~cm}^{-1}\left(\mathrm{C}=\mathrm{O}\right.$ of thiazolidinone ring), $720 \mathrm{~cm}^{-1}\left(\mathrm{C}-\mathrm{S}-\mathrm{C}\right.$ of thiazolidinone ring), $3080-3090 \mathrm{~cm}^{-1}(\mathrm{CH}$ of thiazolidinone ring), 3040-3080 $\mathrm{cm}^{-1}$ (C-H, of Ar.),2950, $1370 \mathrm{~cm}^{-1}\left(-\mathrm{CH}_{2}\right), 1080(-\mathrm{Cl}), 1555,1375\left(-\mathrm{NO}_{2}\right)$. The ${ }^{1} \mathrm{H}$ NMR signal 3.80-3.90 (2H, s,-CH of the ring), 5.94-5.95 $(1 \mathrm{H}, \mathrm{s},-\mathrm{CH}), 6.32-8.44(9 \mathrm{H}, \mathrm{m}, \mathrm{Ar}-\mathrm{H}), 2.54\left(4 \mathrm{H}, \mathrm{t},-\mathrm{CH}_{2}\right), 1.55-$ $1.62\left(6 \mathrm{H}, \mathrm{m}, \mathrm{CH}_{2}\right)$. The $\mathrm{C}, \mathrm{H}, \mathrm{N}, \mathrm{S}$ analysis data of all compounds are shown in Table- 3 .

The assessment of elemental analytical data discloses that the elemental contents are consistence with the expected structure shown in Scheme-1. The IR data also express for assignment of the predicted structure. The final structure of all compounds is confirmed by LC-MS. LC-MS data of all compounds are presented in Tables-1,2 and 3.

\section{CONCLUSION}

The reaction of 4-Amino-1,2,4-triazole (I) with 5-Arylfuran-2-carbaldehydes (IIa-e) yields Schiff bases of N-[(5Arylfuran-2-yl)methylene]-4H-1,2,4-triazol-4-amine (IIIa-e), which on reaction with Thioglycolic acid yielded 2-(5Arylfuran-2-yl)-3-(4H-1,2,4-triazol-4-yl)thiazolidin-4-one (IVa-e) and their Mannich base products (Va-e), their structured were proved by the elemental and spectral analysis. Newly prepared compounds were shows moderate to good antibacterial and antifungal activities.

\section{ACKNOWLEDGEMENT}

The authors are thankful to Department of Chemistry, Rabindranath Tagore University, Bhopal, (M.P.) for providing laboratory facilities.

\section{REFERENCES}

[1]. Shalini Bajaj, Partha Pratim Roy and Jagadish Singh, (2017) Anti-Cancer Agents in Medicinal Chemistry, 17, 1869-1883

[2]. H. Ibrahim Ugras, Ismet Basaran, Turgut Kilic, Umit Cakir, (2006) J. Heterocyclic Chem., 43, 1679.

[3]. Ram U. Ambhure, Sunil R. Mirgane, Devidas U. Thombal, Rajesh B. Nawale, Rajendra P. Marathe and Rajendra P. Pawar, (2017) Mod. Org. Chem. Res., 2(1).

[4]. Shah Alam Khan, Priyanka Ahuja and Asif Husain, (2017) J. Chinese chemical society,64(8),918.

[5]. Purvesh J. Shah, Hasmukh S. Patel, Bhupendra P. Patel, (2013) Journal of Saudi Chemical Society, $17,307$.

[6]. Y. Yi, X. Q. Wei, M. G. Xie, and Z. Y. Lu, (2004) Chinese Chemical Letters., 15(5), 525.

[7]. Purvesh J. Shah, (2016) Heterocyclic Letters, 6 (1), 111.

[8]. Laith Q. Al-Mawsawi, Raveendra Dayam, Laleh Taheri, Myriam Witvrouw, Zeger Debyser and Nouri Neamati, (2007) Bioorg. Med. Chem. Lett. 17(23), 6472.

[9]. Wolfgang Hanefeld, Martin Schlitzer, Norbert Debski, Helmut Euler, (1996) J.Heterocycl.Chem.,33,1143.

[10]. S.Aslam, N.Asif, M. N.Khan, M.A.Khan, M.A.Munawar and M.Nasrullah, (2013) Asian J. of Chemistry, 25(14),7738.

[11]. B. Shivarama Holla,, B. Sooryanarayana Rao, K. Shridhara, P.M. Akberali, (2000) IL Farmaco, 55,338.

[12]. Rudolf Kada, Dušan Ilavský, Jarmila Štetinová, Lubomír Zalibera and Jiř́ Pad’our, (1994) Collect..Czech.. Chem. Commun., $59,444$.

[13]. Christian S. Rondestvedt, Jr, (1960) Org.Reactions, 11, 190.

[14]. Kodihalli C. Ravindra, Hosadu M. Vagdevi and Vijayvithal P. Vaidya., (2008) ARKIVOC 2008 (xi) 1-10. 